\title{
Students' preinstructional reasoning with the speed of light in relativistic situations
}

\author{
Floor Kamphorst $\odot,{ }^{1,2, *}$ M. J. Vollebregt, ${ }^{1,3}$ E. R. Savelsbergh, ${ }^{1,4}$ and W. R. van Joolingen ${ }^{1}$ \\ ${ }^{1}$ Freudenthal Institute, Utrecht University, P.O. Box 85170, 3508 AD Utrecht, the Netherlands \\ ${ }^{2}$ Gemeentelijk Gymnasium Hilversum, Vaartweg 54, 1217 SV Hilversum, the Netherlands \\ ${ }^{3}$ St. Bonifatius College, Burgemeester Fockema Andrealaan 7-9, 3582 KA Utrecht, the Netherlands \\ ${ }^{4}$ Utrecht University of Applied Sciences, Research Centre for Learning and Innovation,
} P.O. Box 14007, 3508 SB Utrecht, the Netherlands

(Received 8 May 2019; published 3 September 2019)

\begin{abstract}
Special relativity theory (SRT) has recently gained popularity as a first introduction to "modern" physics thinking in upper level secondary physics education. A central idea in SRT is the absolute speed of light, with light propagating with uniform speed relative to the reference frame of the observer. Previous research suggests that students, building on their prior understandings of light propagation and relative motion, develop misunderstandings of this idea. The available research provides little detail on the reasoning processes underlying these misunderstandings. We therefore studied secondary education students' preinstructional reasoning about the speed of light in a qualitative study, probing students' reasoning through both verbal reasoning and drawing. Event diagrams (EDs) were used as a representational tool to support student reasoning. Results show that students productively use EDs to reason with light propagation. In line with previous research, we found two alternative reference frames students could use for uniform light propagation. Most students show a flexibility in their use of reference frame: They not only evaluate light propagation in their preferred frame of reference, but also relative to other frames. Some students experienced conflict between an alternative reference frame and the speed of light and changed their reasoning because of that. This finding suggests promising directions for designing education.
\end{abstract}

DOI: 10.1103/PhysRevPhysEducRes.15.020123

\section{INTRODUCTION}

Internationally, the interest for introducing modern physics in secondary school is growing. Novel teaching approaches about topics such as quantum mechanics, nanoscience, and Einstein's theories of relativity, both special and general, have been developed and included in national curricula [1-4]. This is also the case for the Netherlands, where the modern topics quantum mechanics, particle physics, and special relativity theory were introduced in the final years of preuniversity level secondary physics education.

These modern physics topics are characterized by their mathematical complexity, their lack of daily life reference, and their often counterintuitive concepts and consequences. Students have not yet mastered the formal techniques to solve meaningful quantitative problems. This favors a conceptual approach in secondary schools. However, to gain a conceptual understanding, students need to adopt highly formal and radically new frameworks. At the same time, students

*f.kamphorst@uu.nl

Published by the American Physical Society under the terms of the Creative Commons Attribution 4.0 International license. Further distribution of this work must maintain attribution to the author(s) and the published article's title, journal citation, and DOI. cannot resort to life world experiences. Therefore, in modern physics, conceptual learning might prove to be even more difficult than it is for classical physics topics, where students do have direct life world experience with the phenomena at hand (see, e.g., Ref. [5]).

A fundamental premise in conceptual learning is that new conceptual knowledge builds on previous mental structures (concepts, experiences; see, e.g., Refs. [6,7]). Therefore, the introduction of new concepts needs to be closely connected to students' prior thinking [8,9]. It is thus essential to acquire a detailed image of students' ideas and prior thinking related to principles and concepts of these modern physics topics as well. A continued interest in studies with the focus on students' difficulties or misconceptions and students' mental models illustrates this need (cf., $[10,11]$ ). With this study we aim to contribute to these efforts for the topic of special relativity theory (SRT).

A main reason to include a modern subject such as SRT in secondary education is that it is fundamental to modern physics, and an excellent example of a major paradigm shift in the thinking of physicists. From thinking in terms of an absolute space and time, physics moved towards thinking in a combined spacetime with an absolute speed of light, the properties of which depend on the relative motion of observer and observed phenomena. Basic concepts such as simultaneity, space, and time required new definitions. 
SRT is a theory of counterintuitive concepts and consequences. The theory is based on two postulates. The relativity principle states that all laws of nature are the same for observers moving at a constant speed relative to each other. The light postulate states that "Light is always propagated in empty space with a definite velocity $c$ which is independent of the state of motion of the emitting body" [12]. Combined with the relativity principle, this postulate implies that if two observers are in relative motion to each other, and both look at the same traveling light phenomenon, each will see the light travel at the same speed $c$, each relative to their own point of view. This speed is referred to as the absolute speed of light. ${ }^{1}$ The concept of absolute speed by itself may seem counterintuitive, but its implications might be even more counterintuitive from a classical point of view: two observers moving relative to each other will observe different values for the distance the light has traveled and for the time that passed by between two events. This means, for instance, that a clock moving at a certain speed will run slower than one at rest relative to the observer, and the length of the moving clock in the direction of movement will be smaller than the length of a clock at rest relative to the observer.

SRT is difficult to learn for students. The consequences of SRT only become apparent at high speeds. Therefore, we do not have any daily life references for relativistic phenomena. When SRT was first introduced, physicists struggled with the shift towards a combined spacetime, and it should not be surprising that today's students also struggle with the basic relativistic concepts after their first SRT courses [13-15]. The struggle with relativistic concepts might be rooted in problems with the postulates, since all relativistic concepts are derived from them. Several studies have addressed the relativity principle in both classical and relativistic physics $[16,14]$. However, students' understanding of the light postulate is less well documented, especially at the secondary education level. We expect that these students will also struggle to understand the light postulate in view of their prior understandings of light propagation, and that it might be helpful for teaching to gain more insight into their prior understanding.

Young children often do not clearly distinguish between light and its source or effect. From the age of 13 or 14, children more often recognize light "as a distinct entity, located in space between its source and the effect it produces" [17]. Once children come to see light as a distinct entity in space, they still consider light propagation and light travel time only in the context of very long distances, for instance, the distance between Earth and the Sun. In the context of their own environment, light

\footnotetext{
${ }^{1}$ According to SRT, the speed of light is absolute. This means it is constant (does not vary over time), it is uniform (equal in all directions and at all points in space), and it has the same value to any observer, regardless of whether the observer is stationary or moving relative to the light source.
}

arrives instantaneously [17]. Since SRT is taught in the final years of upper level secondary education, we expect these students to be able to reason with light as an "entity in space." However, relativistic reasoning requires students not only to reason with light as an entity in space, but also to apply a notion of propagation time of light even in contexts with small distances, thereby acknowledging that light has a finite speed. ${ }^{2}$

At the upper end of the educational spectrum, Villani and Pacca [18] studied physics graduate students' ideas about the speed of light after they had completed courses on SRT. Most students, even at this advanced level, were committed to the idea that relativistic effects are only apparent. They thought that there is only one true value for the speed of light, and that this true value is measured relative to the light source, independent of the observers' reference frame. If even these graduate students do not reason along the lines of the light postulate, we cannot expect that introducing the light postulate by offering a definition, an approach also adopted in many secondary school books, will lead secondary education students to reason along the lines of the light postulate.

Secondary education students' ideas about light propagation in relativistic contexts prior to instruction are not well documented. However, the study by Dimitriadi and Halkia [3] of secondary education students' use of the light postulate in thought experiments after instruction gives some insight into student talk about light propagation. In this study, some of the student's statements sounded similar to the light postulate, while at the same time suggesting variable speeds relative to the observer. We can illustrate this with a student quote: "Light always has the same speed. What the observers measure... well, this depends on how fast they are going." [3]. From a physicist's perspective, the student quote seems inconsistent: the student first acknowledges the light postulate and then denies it in the same sentence. But if we assume that the learner tries to maintain a coherent worldview [19], the student statement is consistent with the idea that light has a constant speed relative to an absolute space to which all observers agree. In fact, Dimitriadi and Halkia also conclude that students prefer to describe motion relative to Earth, which can also be seen as a kind of absolute space. This dissonance between physics theory and student ideas is also mentioned in the research of Hewson, who found that at first glance university students accept relativistic concepts, such as the light postulate, but that further inquiry reveals they do not understand them in the intended way [20].

In the studies of Dimitriadi and Halkia [3] and Villani and Pacca [18], the underlying reasoning processes remained mostly invisible, which makes full interpretation of students' statements hard. For instance, the student quote

\footnotetext{
${ }^{2}$ As opposed to an infinite speed of light, which will result in an instantaneous arrival of light.
} 
in the previous paragraph shows that statements about a constant speed of light cannot be interpreted without knowing the reference frame relative to which this constancy holds for the student. Thus, when assessing student ideas on light propagation, this needs to be done in a fashion that makes the reference frame in which students do reason explicit. As light propagation always involves space and time, drawings might be a helpful means to have students make the temporal and spatial aspects of their reasoning explicit [21]. Therefore, using drawings in addition to verbal reasoning seems a promising approach to discover the subtle differences between students' and physicists' reasoning and to bring ideas to light that might prove helpful in the teaching and learning of the light postulate.

In the literature, we identified the event diagram (ED) as a suitable instrument to make the reasoning process visible and to elicit students' implicit usage of a reference frame [22]. An ED represents something happening as a series of snapshot pictures, showing the positions of objects at subsequent moments in time. The ED thus represents a series of events in spacetime as seen from a specific reference frame, and different EDs can be used to present the same series of events from different frames of reference. We expect that, in addition to being a powerful tool for teaching [23], EDs can also be a tool for eliciting student reasoning, if students are to construct light propagation by drawing in these diagrams by themselves.

Like in a time lapse movie, time in the ED has a discrete character. Students need to reason stepwise to reach their conclusion. This approach differs markedly from the more usual representations, where only the end or the beginning of the series of events will be mentioned explicitly. Thus, by introducing EDs, it might be expected that the reasoning task alters in a qualitative way. Therefore, we are also interested in the contribution that EDs make to student reasoning, their potential as an evaluative tool, as well as a didactic resource.

To summarize, the literature shows that students' interpretations of the light postulate can be problematic indeed. Rather than using an absolute speed relative to the observers, students were found to reason with a uniform speed of light relative to absolute space [3], or relative to the light source [18]. Although this is a valuable starting point, both studies describe student answers without the underlying reasoning, and they focused on postinstruction reasoning. To teach the light postulate more effectively, we need a more detailed understanding of how secondary education students construct light propagation and uniform speed of light prior to instruction. This qualitative study aims to elicit students' reasoning in a step-by-step fashion, using EDs, to identify elements in their reasoning that might inform our design of SRT education. We therefore ask the following research question: Using event diagrams, in what ways do secondary education students reason with light propagation in relativistic situations prior to instruction?

\section{METHOD}

To answer our research question, we conducted a clinical interview study, following the approach described by Ginsburg [24]. In this approach, the researcher presents a task to the participant and invites the participant to reflect on the task. The researcher then asks further questions, contingent on the participants' responses. This process allows verbalization on the student's part, exposing underlying cognitive processes. The flexible nature of the clinical interview allows the researcher to probe student reasoning until the participant has given a complete answer.

\section{A. Participants}

The 16 participants were 11th grade students of preuniversity secondary education in the age range of 15 to 18 years, from two different schools. The researcher or their physics teacher approached participants in class with an open invitation. Initially more boys volunteered, after which girls were invited more explicitly a second time. Because of a failed audio recording, the final analysis included data from 15 participants (ten boys and five girls).

The participants in this study were all enrolled in the science track. Both schools taught SRT as part of physics education, but the participants had not received any education on this topic prior to the study. Electromagnetic radiation and the wave-particle behavior of light had been discussed earlier in the school year.

\section{B. Procedure and materials}

The interview consists of three phases: an introduction, a nonrelativistic task (A), and a relativistic task (B), each consisting of three subtasks. The participant is asked to think aloud, to express their reasoning in the ED, and to explain what will happen based on their drawings. This way, we use three perspectives to probe student reasoning: doing, describing, and explaining. If the coherence between these three perspectives is not apparent to the researcher, the participant is asked to elaborate. At the end of each phase, the researcher summarizes the main insights that have been addressed to ensure each participant has the required information at the start of the next task.

\section{Introduction phase}

The aim of the introduction phase is to check whether the participant understands the basics of seeing objects and the speed of light. The researcher presents a concept map (Fig. 1) and the participant is asked to talk about light. Subsequently, the researcher asks for examples of situations in which one would notice that light has a speed and to explain why one can see a pencil that is lying on the table. 


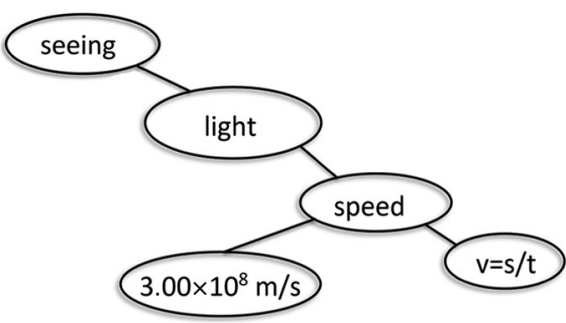

FIG. 1. The light concept map to support students talking about the basics of seeing and the speed of light.

\section{Reasoning tasks $A$ and $B$}

Reasoning task A and B consist of three subtasks each. All (sub)tasks are about physically the same situation: a light flash is emitted by a lamp in a room. At each side of the room, there is a door connected to a light sensor. When the light strikes a light sensor, the door at that side of the room will open. In task A, the observer has a fixed position relative to the room with each subtask having the observer in a different position. In the subtasks for task B, the observer and/or the room are moving, relative to each other or relative to the paper.

The reasoning tasks are presented verbally and illustrated with EDs. At the start of the interview, the researcher briefly explains the way time and motion are represented in EDs, that light has a speed of two squares per time unit in these diagrams, and how to draw light in the EDs. After this instruction phase, the participant is free to choose a drawing method that suits them best.

At the start of each subtask, the researcher describes the situation and asks the participant to take the perspective of the observer. Each time the participant is asked the following:

(1) At what time do the doors open after the light flashes?
(2) When will the observer see this happen?

(3) Do the doors open simultaneously?

(4) Does the observer see the doors open simultaneously?

If a participant has trouble coming up with an answer, the researcher stimulates the student to use the ED in constructing their answer. Once the participant has completed all three subtasks of task A or B, they are asked to compare the task outcomes. The researcher asks participants to compare their answers of the different subtasks and explain why the EDs lead to different or identical answers. The participants are also asked to compare subtask B3 to subtask A1.

The EDs used in this study present the passing of time using sequences of 6-12 pictures representing the layout of the situation at subsequent moments in time. The first instant is shown at the top of the page, the last at the bottom. Each picture is drawn on graph paper and shows the position of the lamp, the room, and the observer. The propagation of the light is not shown in the pictures: it must be constructed by the participant themselves. Figures 2 and 3 present the first pictures for each subtask of task A and $\mathrm{B}$, respectively.

The aim of task A is to probe whether participants reason with a constant speed of light in the ED, and whether they consider signal travel time. In subtask A1, both the lamp and the observer are in the middle of the room. This means that light will reach the sensors at both ends of the room simultaneously and that the observer will see the doors open simultaneously as well. In the ED, applying a two squares per time unit speed of light, the doors will open at $t=3$, and the observer will see this happen at $t=6$. In subtask A2, the lamp is still in the middle of the room, which means that light reaches the sensors simultaneously at $t=3$. However, the observer is positioned closer to the left-hand door, which means that they see the left-hand
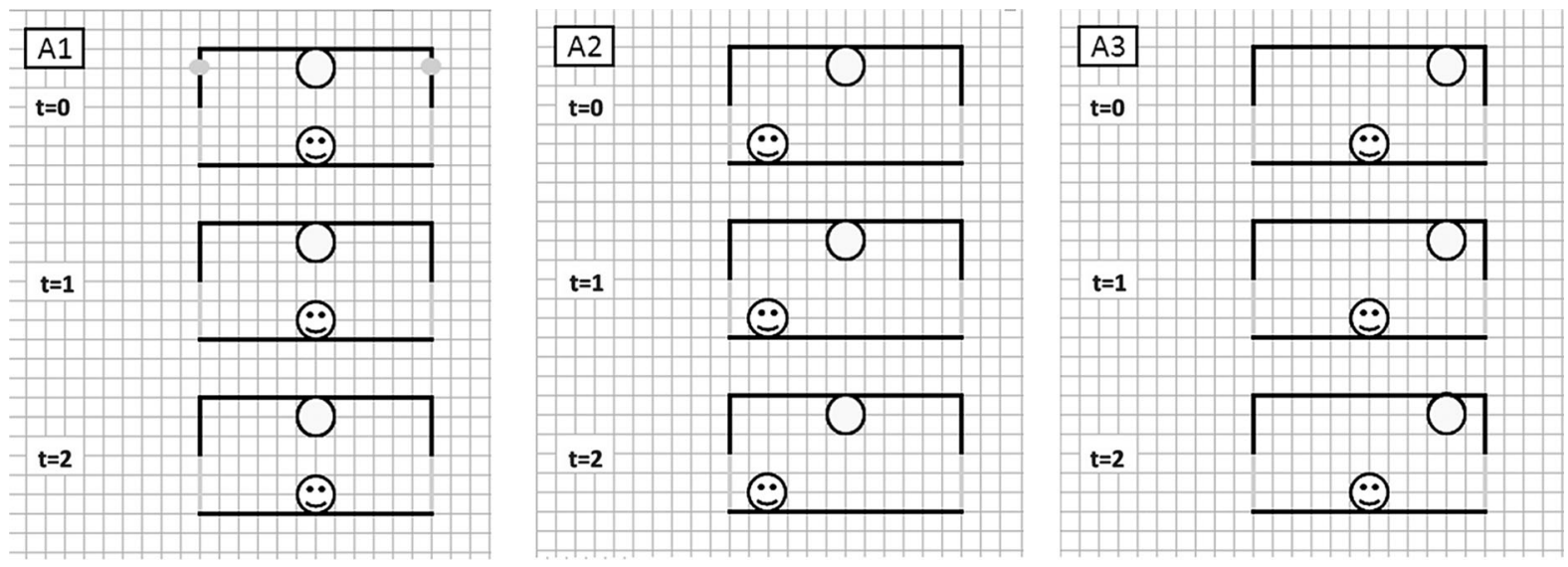

FIG. 2. Task A. EDs to probe reasoning with signal travel time and correction for signal travel time. In the diagrams, the room is depicted as a rectangle, the light source is the circle at the top, the doors are the lines at the side of the rectangle, and the observer is represented by the smiley face. To prevent an overcrowded ED, the sensors, the dots above the doors, are only shown in the first picture of task A1. Participants are instructed that the sensors are present in all the tasks. 

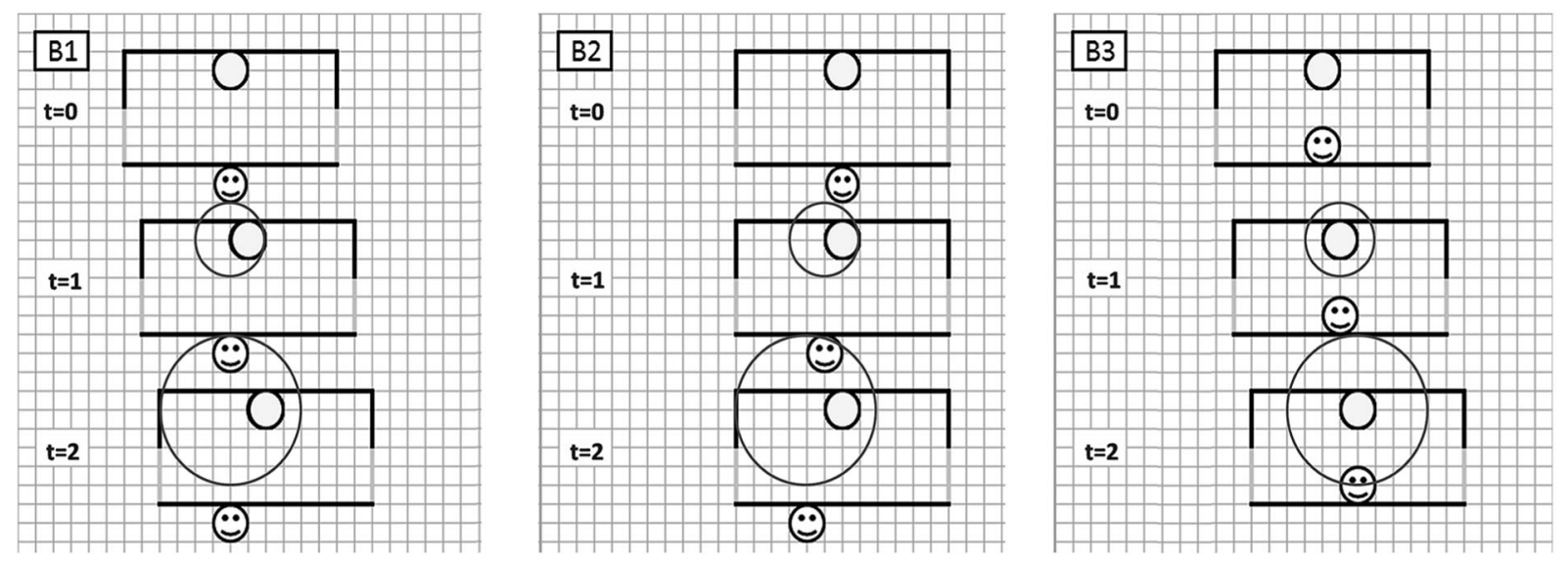

FIG. 3. Task B. EDs to probe the reference frame in which participants think light propagation is uniform. The gray circle represents the correct answers according to SRT. These were not included in the version given to the students.

door open first at $t=4$ and the right-hand door open at $t=8$. In subtask A3, the lamp is positioned closer to the right-hand door. As a result, the doors will not open simultaneously. The right-hand door opens at $t=1$ and the left-hand door at $t=5$. The observer is still in the middle of the room and sees the doors open at $t=4$ and $t=8$, respectively. We expect that participants who reason with a constant speed of light will draw light that covers equal distances for each time interval in the ED. Participants who consider signal travel time will say that the observer sees the doors open a while after the event has happened. On the other hand, participants who do not reason with a finite speed of light will say that the doors open instantaneously.

Task B is designed to clarify how students conceptualize the constant speed of light in situations where the lamp has a relative speed to the observer. In subtask B1, the room is moving relative to the graph paper and the observer. If students reason with a constant speed of light relative to the observer, they will conclude that light reaches the sensor for the left-hand door first at $t=2$ and that the observer will see this at $t=4$. The right-hand door will open at $t=6$ and the observer sees this at $t=12$. The student will conclude that the doors do not open simultaneously, and that the observer will not see this happen simultaneously either. However, if a student reasons with a constant speed of light relative to the light source, they will conclude that both doors open simultaneously at $t=3$. In subtask B2, the observer is moving relative to the graph paper and the room. Students who apply the light postulate will obtain the same task outcome as in task B1. However, if students do not apply the light postulate and reason with a constant speed of light relative to the lamp or the graph paper instead, they will conclude that both doors open simultaneously at $t=3$. Subtasks B1 and B2 will give an initial idea of whether a student reasons with an absolute speed of light or an alternative reference frame. Subtask B3 will give more clarity of which alternative reference frame this might be. In this subtask both room and observer move relative to the graph paper. Students who reason with a constant speed of light relative to the lamp will obtain the same answers as in subtask A1, whereas students who reason with a constant light speed relative to the graph paper will obtain similar answers as in subtask B1.

\section{Data collection}

Participants were interviewed individually for 4560 minutes. We videotaped the interviews and we collected the event diagrams participants produced. Interviews were transcribed verbatim. Comments for clarification are placed within square brackets.

\section{Analysis and quality assurance}

To obtain a full account of student reasoning, we used an iterative approach in which all data sources (video, transcript, and EDs) were analyzed together. We followed the method of interpretative analysis described by Clement:

\section{Essentially, the scientist aims to construct or piece together a theoretical model in the form of a conjectured story or a picture of a hidden structure or process that explains why the phenomenon occurred. [...] the initial model is evaluated and revised in response to criticisms. [...] In this method, analysts construct, criticize, and revise hypothesized models of mental structures and processes repeatedly while using them to explain as much of the data in a protocol or a set of protocols as possible [25].}

Our analysis is composed of two phases. In the first phase we familiarized ourselves with the data. For part of the data (7 students), characteristic student quotes and task outcomes that were indicative of the use of a particular reference frame were coded in an open fashion. For example, the student quote "so here at $\mathrm{t}$ is zero, [the light] is still in the lamp itself. At time one [the light] is two 
squares further ... At time three it is again two further [...] So eventually $[\ldots]$ in this room [B1], the light arrives at both sensors at the same time and so you see that both doors, they open at the same time." (Nick) was coded with "doors open simultaneously, B1." The drawing showed that Nick counted the two squares relative to the lamp. Therefore, the drawing, together with the quote, was also coded with "relative to lamp." These coded segments of all tasks together formed the input to interpret student reasoning. If a student would consistently apply the same reference frame for light propagation over all subtasks, this person could be classified as holding that particular view on uniform light propagation.

We created a holistic narrative of each participant interview. This was a chronological description of the interview, which focused on what participants said and drew about light, the speed of light, light propagation, and task outcome. The narratives illustrate how participants explain that reasoning pattern (interpretative analysis [25]).

The second phase covers the entire dataset. The data were coded and interpreted as described above. In the end we grouped the students with similar reasoning patterns and looked for similarities within these groups and differences between the reasoning patterns (thematic analysis [26]). If participants did not fit one of the categories at first glance, they were discussed in the research team until consensus about their reasoning was reached.

\section{RESULTS}

We will first discuss how participants worked with the EDs and if the tasks indeed triggered student responses that were in line with the aim of the task design. Subsequently, we will discuss the alternative reference frames students have when they reason with light propagation in relativistic tasks, and the results of task B will be discussed more in depth.

\section{A. Introduction phase}

The aim of the introduction phase was to check whether participants understood the basics of seeing objects and speed of light. All participants mentioned that the speed of light is constant.

Nonrelativistic task A aimed to familiarize students with the reasoning task and the EDs. Participants did not express difficulty with the reasoning task itself. The nonrelativistic task could easily be solved by measuring the distance light had to cover in the ED and then applying basic reasoning with longer distances taking longer time for light to cover.

Before the researcher instructed participants how to draw light propagation in the ED, participants used the EDs in three different ways. Participants either only drew the light emitted in the first picture $(t=0)$ [Fig. 4, left-hand ED $(7 / 15)$ ] or tried to fit all events in one picture [Fig. 4, middle ED (4/15)]. These participants did not use the time dimension of the ED, even though they had a chronology of the events in explaining their drawing. A few participants $(3 / 15)$ did use the discrete time dimension of EDs spontaneously. They drew the course of events spread out over three separate pictures (Fig. 4, right-hand ED). One participant did not draw in the ED.

After instruction on how to use EDs, all participants could draw light propagation by using the time dimension of the EDs. All participants drew light that propagated at a constant speed in task A. An example is shown in Fig. 5. The dot and circle in the first two pictures were drawn by the researcher; the other circles were drawn by
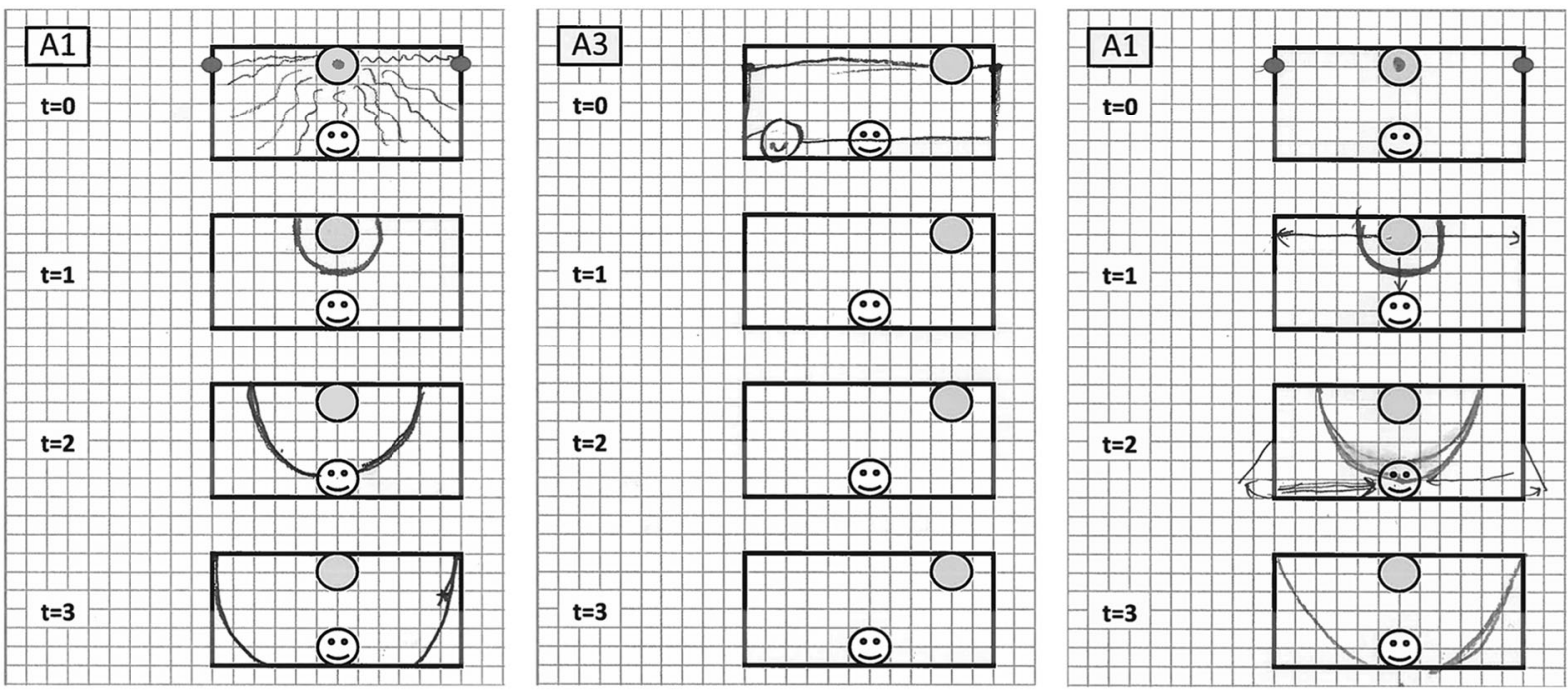

FIG. 4. Participants used EDs in several ways. Light going in all directions (left-hand ED), indication of the path light covers (middle ED), and individual time units for different events (right-hand ED). The semicircles represent wave fronts. How to draw wave fronts is part of the instruction on working with EDs in subtask A1. 

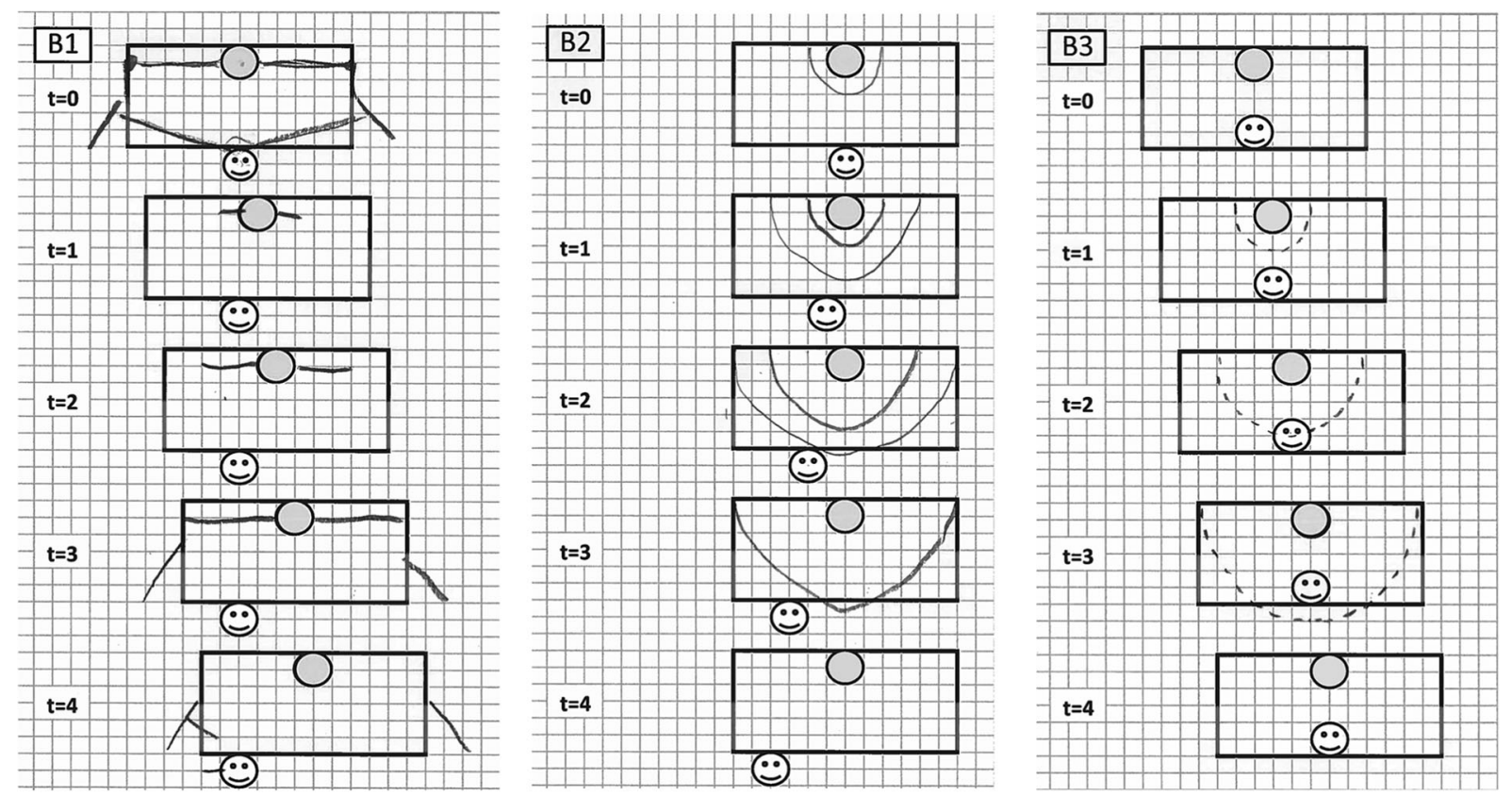

FIG. 5. EDs of participants who drew uniform light propagation relative to the lamp. The position of the light flash changes with two squares relative to the position of the lamp. The ED on the left shows the drawing of a participant who indicated the distance the light has covered as a path length. The other participants drew wave fronts.

the participant. After instruction, participants were free to choose their own way of drawing light in the ED. We did not see any relation between drawing method (wave fronts, horizontal lines, dots) and reference frame for a uniform speed of light.

The aim of task B was to find out if students used an alternative reference frame for light propagation. To solve task B students had to use the ED. Fourteen out of 15 students could work with the ED in the intended way, constructing their answer step by step and using the time dimension of the ED to their advantage. One student kept measuring distances and ignored the time dimension of the ED. Although she received explicit instruction how to use the diagram, she did not work with it in these relativistic tasks. We therefore could not use her data to assign a reference frame for uniform light propagation. For two participants, the wave front drawings raised unhelpful associations about Doppler shift, blue shift and red shift of starlight, and water waves in front of a boat. These associations made the task more difficult for the students, but they still managed, so their data were included in our analysis of task B.

All participants adopted stepwise reasoning approaches, as illustrated by this quote from Kevin:

Kevin: It [the light] will be emitted here.... Then it will ... let me think ... but yes, I think just two squares further ... so here... there.

At each step, participants would determine the distance the light had moved since the last time unit, thus determining the position of the wave front for each time unit in the ED. In this, a combination of reasoning with distance and determining the position of the light flash in the ED was used.

\section{B. Alternative reference frames for uniform speed of light}

As discussed above, 14 participants could work with the relativistic subtask B. In the following section we will discuss the line of reasoning of these 14 students more in depth.

All 14 participants took a similar approach to task B: they drew wave fronts and determined the position of the light flash in the ED. However, for some participants, the wave front did expand at a uniform speed relative to the lamp in all three situations (Fig. 5). For others, the wave front did expand relative to a fixed point on the paper (Fig. 6). From a physics point of view these viewpoints could be interpreted as the reference frame of the lamp and the reference frame of the graph paper, respectively. These reference frames did not coincide with the observer in all situations. So, although participants were asked to reason from the perspective of the observer, they drew uniform light propagation in a different reference frame. We will refer to this as "reasoning in the frame of the lamp (or graph paper)."

Some participants started out reasoning in the reference frame of the lamp and switched to the reference frame of the graph paper at some point during the task. Furthermore, one participant showed characteristics of both frames throughout his reasoning. 

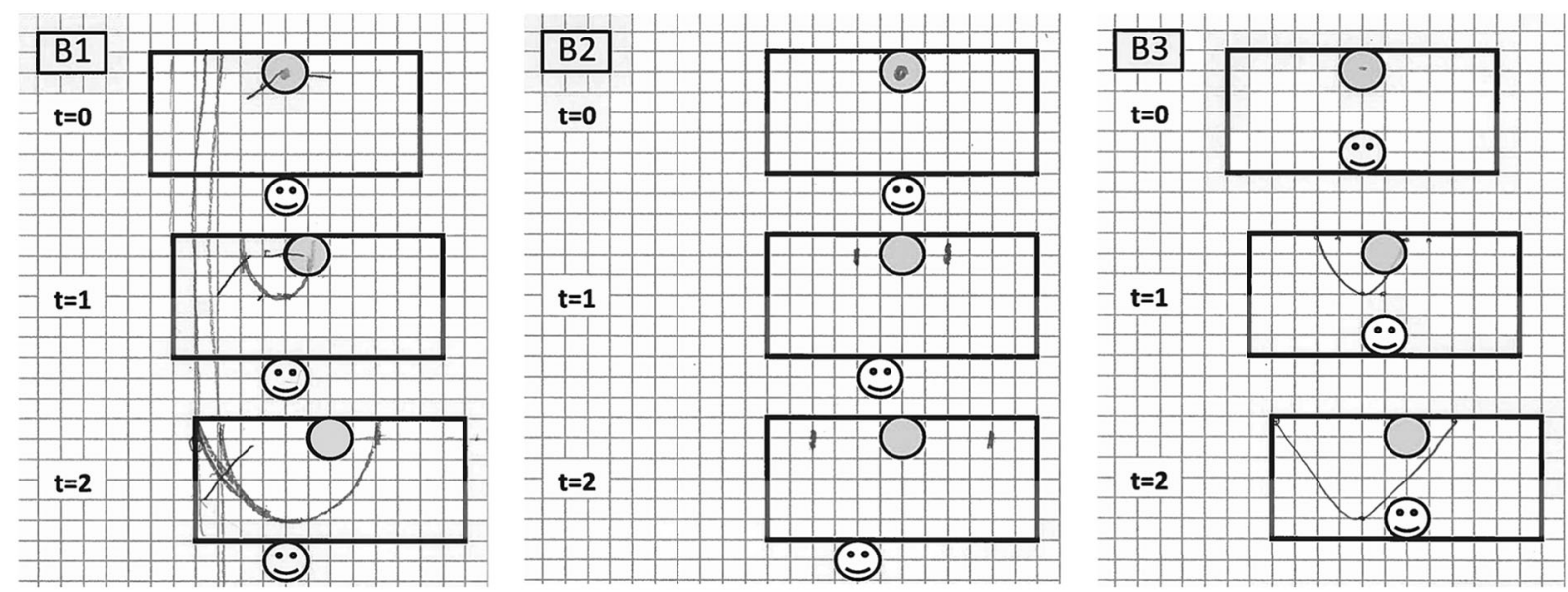

FIG. 6. EDs of participants who drew uniform light propagation relative to the graph paper. The position of the light flash changes each time step with two squares relative to the position on the paper the light flash had in the previous time unit. The ED in the middle shows that the participant only indicated the position of the wave front in the horizontal direction. The other participants drew wave fronts.

Finally, one participant could not be placed in this dichotomy: she drew a uniform speed of light relative to the observer. This participant explained her reasoning referring to relativity theory and the light postulate. As it turned out during the interview, this participant had extensively studied special relativity as a personal side project. Therefore, her reasoning is not representative of reasoning teachers could expect in their classrooms. However, this participant showed that the task design is also suited for reasoning along the lines of SRT. An overview of the different reference frames and participants is given in Table I. Not only did these groups think differently about light propagation, they also had a different interpretation of how movement in the task should be described.

\section{Reference frame lamp and room}

Participants who drew light with a uniform speed relative to the lamp said the doors did open simultaneously in all situations. The following quotes illustrate typical arguments, solely based on the geometry of the situation:

Tim: For the doors open at the same time in B1, as well as B2 [and] B3, because the lamp is, I think, just in the middle of the room.

TABLE I. Number of participants per reference frame for uniform light propagation.

\begin{tabular}{lc}
\hline \hline Reference frame & $\begin{array}{c}\text { Number of } \\
\text { participants }\end{array}$ \\
\hline Observer & 1 \\
Lamp & 4 \\
Graph paper & 4 \\
Change: lamp $\rightarrow$ graph paper & 4 \\
Combination: lamp and graph paper & 1 \\
\hline \hline
\end{tabular}

Anouk: Actually, ehm, in each situation here [B1, 2 and 3], exactly the same thing will happen. So, the light will turn on, and ehm the doors open at exactly the same moment because [the lamp] is exactly in the middle [of the room]. [...] So ehm the light will just ehm move forward with a certain speed. Even if the observer or the box is moving.

For students taking this approach, all that mattered were relative positions. The relative movement of the room and observer did not influence light propagation for students with this mode of reasoning.

In principle, the observed reasoning patterns can be explained by participants reasoning with a constant speed of light relative to the lamp or relative to the room. In our task design, the lamp and room were always stationary relative to each other, so we cannot clearly distinguish between these frames, and we will call this reference frame the lamp-room frame from now on.

\section{Reference frame graph paper}

Participants who drew light with a uniform speed relative to the graph paper said that the doors would open simultaneously in subtask B2, but in subtasks B1 and B3 the lefthand door would open first.

Participants were focused on what is moving in the situations when they oriented themselves on the tasks:

Kevin: Does the room move here, or the person?

Niels: [...] but does the room, does it move to the right, or does the observer move to the left?

When asked whether it mattered if the room or the person would move, the participants answered affirmative. Participants concluded that the room moves in situations 
$\mathrm{B} 1$ and $\mathrm{B} 3$, and the observer moves in $\mathrm{B} 2$; all this movement is described relative to the graph paper.

When explaining the outcomes of subtasks B1 and B3, participants would focus on movement of light and objects in the ED again:

Thomas: The light is emitted from the lamp and before it reaches the sensors, the distance decreases. So, the required time [for the light to reach the sensors] decreases.

Kevin: Well, ehm, because [...] the room moves, the light will ehm, move in the same way, but the room with the lamp will move further to the right. So it will, at some moment it will ehm ... it seems as if the light is more to the left of the lamp than it actually is.

According to Thomas, light had a shorter distance to cover because the room was also moving (relative to the graph paper). Kevin used the same line of reasoning, but also expressed that light had covered a bigger distance relative to the lamp than what one would expect in a nonrelativistic situation. Kevin did not express anything that would suggest that he thinks this bigger distance contradicts with a constant speed of light.

To these participants subtask B2, where the room is at rest relative to the paper and the observer is moving, is rather different from B1. Even though the relative motion between the lamp and the observer is the same:

Kevin: In this situation [B2], the doors will open simultaneously, [...] The light will reach the sensors simultaneously because it [the room] is standing still and not moving.

Thomas: With B1 [...] the room moves, with B2 the person moves. That is why with B1 ehm, the doors do not open simultaneously, and with B2, ehm, because the room is standing still, the doors do open simultaneously.

\section{Change of reference frame}

Four participants initially drew light propagation in line with the reference frame of the lamp-room frame. During the interview, they changed their answers, clearly stating the previous answers were wrong. They proceeded with the task by drawing uniform light propagation in the reference frame of the graph paper. We will refer to this process as "changing reference frame."

Two participants changed light propagation frame because they became aware of a different option and found this option more plausible. We will illustrate this with the case of Nick. Initially Nick reasoned with light propagation in the lamp frame in subtask B1:

Nick: To the observer it feels like, the light seems to have gone slower, since [...] the entire room, with the lamp in it, moves away from the observer.
This changed later in the interview, when Nick looked again at subtask B1.

Nick: But...actually, the light has already been emitted, so it just still covers the same [...] distance. [...] This means that we, according to us, the lamp is still above us [...], but the room has moved one square to the right. [...] Because technically, relative to this door [points at the right door], he [the light] moves only one square per second to the right.

This reasoning was consistent with a uniform speed of light relative to the graph paper. Nick explained that light still covered the same distance per time unit when the room moves relative to the observer. In both his answers, Nick mentioned that the speed of light is different when seen from another reference frame. Eventually, that light was emitted at $t=0$ and that the lamp moved away from that point on the graph paper seemed to be the reason that Nick switched to a different reference frame for light propagation. In his answer Nick reasoned also in the reference frame of the observer ("according to us, the lamp is still above us"). But if we look at his drawing in all subtasks, Nick drew uniform light propagation in the reference frame of the graph paper. Furthermore, Nick spontaneously evaluated this new light propagation relative to the lamp-room frame as well: Light moving to the left had a speed of three squares per time unit, relative to the lamp-room frame. When prompted, Nick argued that this answer is not inconsistent with the constant value of two squares per time unit for the speed of light.

Two other participants changed reference frames for light propagation because they experienced conflict between their answer and the speed of light as the maximum speed in nature. We will illustrate this with the case of Sanne. Initially, Sanne drew and reasoned consistently with a reference frame of the lamp-room frame. She explained that, although in subtask B1 the observer moves, and in B2 the room moves, there is no difference between the outcomes of the two subtasks. However, when she started working on subtask B3, she concluded that the moving room did influence the task outcome:

Sanne: OK, it does matter, but I do not really know how. [..] I don't really know how to continue with that.

Sanne proceeded with subtask B3 and still drew light in the reference frame of the lamp-room frame. The researcher asked her to reflect on the results. In this, she evaluated light propagation in the frame of the graph paper, which resulted in a value for light propagation that exceeded the speed of light. This result seemed to confuse her, and she expressed that there is a conflict between her answer and the speed of light as a maximum speed. She repeated three times that this was problematic: 
Sanne: Yes, that cannot be higher, nothing can become higher than the speed of light, at least that is what I have heard all my life, ehm...

Eventually Sanne changed her answer in both subtasks B1 and B3. If Sanne would have adopted the light postulate instead of the paper reference frame, there would still be a conflict between the speed of light as maximum speed and the outcome of subtask B2. Sanne did not express any conflict with this subtask. We therefore conclude that she indeed switched to the paper frame.

\section{Flexible use of reference frames}

Evaluating the speed of light in a different reference frame than the light propagation frame was also observed with six participants who did not change their propagation frame. For these participants, evaluating light speed in other reference frames did not lead to a conflict. We will illustrate this with two examples. Niels uses the graph paper frame and Tim uses the lamp-room frame for uniform light propagation. Both students also evaluate the speed of light relative to the observer:

Niels: Because here [B2, light that goes to the left] you walk along with the speed of light, so relative to you it will go slower. It is the same idea as when you bike along with a car. If you bike away from the car, it will go away faster, for your idea, then when you bike along. So, for your idea light will go faster to the right.

Tim: The light still covers the same distance. Say, in real life, light goes with [...] $3 \times 10^{8} \mathrm{~m} / \mathrm{s}$ and if you also go with $3 \times 10^{8}$, and if you move away from the light with that speed, then you move, and the light moves, but you will never see it, because it will never get closer, or it will never go further away, although you both move [...] Yes, if you leave one second earlier [than the light flash] and you move just as fast, you will never see it.

These results show that students can evaluate the speed of light from several reference frames. However, they stick to one preferred frame where the speed of light should be uniform and equal to the set value of two squares per time unit. This suggests that participants have the tendency to think that movement was more real in one reference frame and just apparent in others. This reference frame coincides with the frame for uniform light propagation.

\section{Combination of two reference frames}

One participant did not fit one of the afore categories. This participant was also focused on what is moving, like participants who reason in the reference frame of the graph paper. However, this participant did not keep the speed of light drawn in the EDs constant relative to one single frame. Light that traveled in the propagation direction of the light source moved with two squares relative to the lamp. The participant explains this by saying that the light is pushed by the light source. Light traveling in the opposite direction had a speed of two squares per time unit relative to the graph paper. The participant said this light is left behind and that the illuminated area is growing.

\section{CONCLUSIONS}

The purpose of our study is to find out in what ways students reason with light propagation in relativistic situations prior to instruction. We found that all but one of the participants could work with EDs. We therefore conclude that students can learn to productively use EDs as a reasoning tool. We found one participant who had already studied SRT, and who reasoned with an absolute speed of light. The other participants reasoned with a uniform speed of light relative either to the lamp or to the graph paper or, in one case, with a mixture of both. We therefore conclude that secondary education students do reason with light propagation in one of two alternative reference frames.

Students consistently used a single preferred reference frame across multiple subtasks. Most importantly, this study showed that many students do not only evaluate the speed of light relative to their preferred reference frame for uniform light propagation, but that they also are able to do this relative to other reference frames. Students are aware that light travels at a different speed as seen from these other frames, but most students do not spontaneously regard this as problematic. Some students did experience a conflict when comparing light propagation in several reference frames, which led them to change their reference frame for uniform light propagation.

\section{DISCUSSION AND IMPLICATIONS FOR EDUCATION}

This study was intended to gain input for designing SRT education and therefore had an explorative character. Although we cannot claim an exhaustive overview of all possible reasoning patterns and their frequencies, we argue that the 15 in-depth interviews did yield new insight into student reasoning on light propagation in relativistic situations and form a sufficient basis for a well-founded design of SRT education.

A possible objection against our findings could be that the reference frames students use for uniform light propagation are induced by our external representation, the ED. EDs are always drawn from one specific reference frame or observer [22]. It is impossible to create EDs that do not favor one reference frame over another. However, we created the EDs in this study to show the same situation in different reference frames. The task design allowed students to describe uniform motion in three reference frames: the graph paper, the room, and the observer. Although the EDs allowed students to draw 
light propagation in a specific reference frame over the subtasks, one of the participants still did not reason with light propagation in one specific reference frame. We therefore conclude that our task design did not force students to use a reference frame for the speed of light at all. Furthermore, our data showed variance in the reference frames participants chose for uniform light propagation. We therefore conclude that the task design did not force students to favor one reference frame over the others.

Previous studies suggest that students do have difficulty describing the same situation from different reference frames, and that these difficulties should be overcome by more or better teaching of Galilean relativity $[15,23,18]$. By contrast, the participants in our sample showed great flexibility in using reference frames. They could describe the speed of the observer, room, and light relative to several reference frames (the paper, lamp, and observer). These results indicate that switching between reference frames itself is not problematic. In contrast to SRT, where the speed of light is absolute, in Galilean relativity the speed of light is different in each reference frame. Therefore, focusing on Galilean relativity alone might not help students overcome their difficulties with the light postulate.

Although both students and physicists may agree that the speed of light is constant, students give a different meaning to these words. It seems as if students think that "the same for all observers" means that all observers will agree on a value for light speed, but that this value is not the same relative to all inertial observers. For physicists, "the same for all observers" does mean that all inertial observers will measure the same value for light speed in their own reference frame. Education should make students aware of this difference between agreeing on the same value and measuring the same value in all inertial frames.

The fact that most students do reason from multiple frameworks, and that some even change their way of reasoning with light propagation, leads us to expect that explicitly problematizing alternative reference frames for light propagation in this way may be a promising approach for teaching the light postulate. EDs support students to make their ideas on light propagation explicit, constructing their ideas step by step, and to reflect on those ideas. Therefore, EDs seem a promising tool to support students in problematizing alternative reference frames for light propagation. Whether or not this approach will also convince students of the light postulate itself is the topic of our next study.

\section{ACKNOWLEDGMENTS}

The authors would like to thank Tienke de Vries for her help with the analysis and all of the students who participated in this study for their time and effort. This work was supported by the Dutch Ministry of Education, Culture and Science under Grant No. OCW/PromoDoc/ 1065001.
[1] E. K. Henriksen, B. Bungum, C. Angell, C. W. Tellefsen, T. Frågåt, and M. V. Bøe, Relativity, quantum physics and philosophy in the upper secondary curriculum: Challenges, opportunities and proposed approaches, Phys. Educ. 49, 678 (2014).

[2] A. Laherto, Incorporating nanoscale science and technology into secondary school curriculum: Views of nano-trained science teachers, Nord. Stud. Sci. Educ. 7, 126 (2011).

[3] K Dimitriadi and K. Halkia, Secondary students' understanding of basic ideas of special relativity, Int. J. Sci. Educ. 34, 2565 (2012).

[4] M. Kersting, E. K. Henriksen, M. V. Bøe, and C. Angell, General relativity in upper secondary school: Design and evaluation of an online learning environment using the model of educational reconstruction, Phys. Rev. Phys. Educ. Res. 14, 010130 (2018).

[5] M. Limon, On the cognitive conflict as an instructional strategy for conceptual change: A critical appraisal, Learn. Instr. 11, 357 (2001).

[6] A. A. DiSessa, What do "just plain folk" know about physics? in The Handbook of Education, and Human Development., edited by D. R. Olson and
N. Torrance (Blackwell Publishers, Cambridge, MA, 1996), pp. 709-730.

[7] A. Shtulman and J. Valcarcel, Scientific knowledge suppresses but does not supplant earlier intuitions, Cognition 124, 209 (2012).

[8] K. A. Strike and G. J. Posner, A revisionist theory of conceptual change, in Philosophy of Science, Cognitive Psychology, and Educational Theory and Practice, edited by R. A. Duschl and R. J. Hamilton (State University of New York Press, Albany, NY, 1992), pp. 147-176.

[9] C. W. J. M. Klaassen, A Problem-Posing Approach to Teaching the Topic of Radioactivity (CD- $\beta$ Press, Utrecht, 1995), pp. 85-110.

[10] F. Savall-Alemany, J. L. Domènech-Blanco, J. Guisasola, and J. Martínez-Torregrosa, Identifying student and teacher difficulties in interpreting atomic spectra using a quantum model of emission and absorption of radiation, Phys. Rev. Phys. Educ. Res. 12, 010132 (2016).

[11] K. Krijtenburg-Lewerissa, H. J. Pol, A. Brinkman, and W. R. van Joolingen, Insights into teaching quantum mechanics in secondary and lower undergraduate education, Phys. Rev. Phys. Educ. Res. 13, 010109 (2017). 
[12] A. Einstein, On the electrodynamics of moving bodies, Ann. Phys. (Berlin) 322, 891 (1905).

[13] J.S. Aslanides and C. M. Savage, Relativity concept inventory: Development, analysis, and results, Phys. Rev. ST Phys. Educ. Res. 9, 010118 (2013).

[14] M. Pietrocola and A. Zylbersztajn, The use of the principle of relativity in the interpretation of phenomena by undergraduate physics students, Int. J. Sci. Educ. 21, 261 (1999).

[15] R. E. Scherr, P. S. Shaffer, and S. Vokos, Student understanding of time in special relativity-Simultaneity and reference frames, Phys. Ed. Res. Am. J. Phys. Suppl. 69, 24 (2001).

[16] S. Panse, J. Ramadas, and A. Kumar, Alternative conceptions in Galilean relativity: Frames of reference, Int. J. Sci. Educ. 16, 63 (1994).

[17] E. Guesne, in Light in Children's Ideas in Science, edited by R. Driver, E. Guesne, and A. Tieberghien (M.A. Thomson Litho Limited, Glasgow, 1989), pp. 10-32.

[18] A. Villani and J. L. Pacca, Students' spontaneous ideas about the speed of light, Int. J. Sci. Educ. 9, 55 (1987).

[19] C. W. Klaassen and P. L. Lijnse, Interpreting students' and teachers' discourse in science classes: An underestimated problem?, J. Res. Sci. Teach. 33, 115 (1996).
[20] P. W. Hewson, A case study of conceptual change in special relativity: The influence of prior knowledge in learning, Eur. J. Sci. Educ. 4, 61 (1982).

[21] S. Ainsworth, V. Prain, and R. Tytler, Drawing to learn in science, Science 333, 1096 (2011).

[22] R. E. Scherr, An investigation of student understanding of basic concepts in special relativity, $\mathrm{Ph} . \mathrm{D}$. thesis, University of Washington, 2001.

[23] R. E. Scherr, P. S. Shaffer, and S. Vokos, The challenge of changing deeply-held student beliefs about the relativity of simultaneity, Am. J. Phys. 70, 1238 (2002).

[24] H. Ginsburg, The clinical interview in psychological research on mathematical thinking: Aims, rationales, techniques, Learn. Math. 1, 4 (1981).

[25] J. Clement, in Analysis of Clinical Interviews: Foundations and Model Viability, in Handbook of Research Design in Mathematics, and Science Education edited by A. Kelly and R. Lesh (Lawrence Erlbaum Associates, London, 2000), pp. 547-589.

[26] V. Braun and V. Clarke, Using thematic analysis in psychology, Qual. Res. Psychol. 3, 77 (2006). 